\title{
Experimentally-aided Simulation of Directional Solidification of Steel
}

\author{
Salar NIKNAFS* and Rian DIPPENAAR \\ University of Wollongong, Northfields Avenue, Gwynneville, NSW 2500, Australia. \\ (Received on September 3, 2013; accepted on January 7, 2014)
}

\begin{abstract}
Dendritic microstructures are most dominant patterns in solidified alloys. The microstructural features of these structures control the segregation profiles of solute elements in the interdendritic regions, thus determining the mechanical properties of cast structures. In this study, a 2D model of solid/liquid interface instability in a low carbon steel was introduced, using the multi-phase-field software code MICRESS ${ }^{\circledR}$ combined with an in-situ study of solidification in a laser-scanning confocal microscope. The use of a moving-frame boundary condition and a linear temperature gradient within the simulation allows further optimization of the solidification studies in the laser-scanning confocal microscope. By analysing the shape of the delta-ferrite grain boundary at the solid/liquid interface, in-situ and at temperature, it was possible to experimentally determine the Gibbs-Thomson coefficient and the solid/liquid interfacial energy of the alloy. The interface mobility of the solid/liquid interface was calibrated in the model so as to reproduce the experimentally measured interface velocity at the onset of interface instability. The proposed model was used to describe the morphological transitions from planar to cellular to dendritic modes during solidification and solute segregation under a variety of processing conditions such as cooling rate and temperature gradient. The importance of this approach is that the verified model has been used to extend the prediction of microstructural development to cooling rates well beyond what can be achieved experimentally and into the regime pertinent to high-speed continuous casting. Significant microstructural differences that arise as a result of varying processing conditions are discussed.
\end{abstract}

KEY WORDS: steel solidification; MICRESS ${ }^{\circledR}$ phase-field; in-situ observations; confocal microscopy.

\section{Introduction}

One of the impediments to the in-situ study of microstructural development of high-melting point metallic systems such as steel during solidification is the difficulty of making observations at the very high temperatures involved. However, experimental techniques have recently been developed that have overcome, at least in part, some of the difficulties of obtaining quality observations at temperature and in real time. Chikama et al. ${ }^{1)}$ were the first to directly observe the planar to cellular and cellular to dendritic transition in $\mathrm{Fe}-$ $\mathrm{C}$ alloys using High-temperature Laser-Scanning Confocal Microscopy (LSCM). They melted the whole sample in an alumina crucible and observed the solid/liquid front during solidification, but their observations were impeded by the presence of a meniscus effect, which rendered high resolution observations difficult. In an attempt to overcome the impediments of meniscus formation, Reid et al. ${ }^{2)}$ developed a so-called concentric solidification technique. By this technique, observations could be made of microstructural devel-

\footnotetext{
This article is one which was originally scheduled for publication in the special issue (Vol. 54, No. 2) on "Cutting Edge of Computer Simulation of Solidification, Casting and Refining" and instead was specially published in this regular issue.

* Corresponding author: E-mail:sn871@uowmail.edu.au

DOI: http://dx.doi.org/10.2355/isijinternational.54.526
}

opment during the solidification of $\mathrm{Fe}-\mathrm{C}$ alloys in real time and at the pertaining temperature. The concentric solidification technique ${ }^{2)}$ consists of creating a melt pool in the center of disc-shaped, typically $250 \mu \mathrm{m}$-thick samples, while retaining a delta-ferrite rim around the melt pool. Using this concentric solidification technique, the meniscus effect is eliminated and a significantly larger area can be observed at high resolution and hence, the planar to cellular to dendritic transitions can be made at high resolution. ${ }^{2)}$

The ability to observe in-situ the microstructural development and the solidification front at temperatures as high as $1870 \mathrm{~K}$ provides a new technique and an enhanced capability to the study of solidification in metallic systems compared with conventional directional solidification experiments such as Bridgman-type furnace studies. A disadvantage of solidification studies using the concentric solidification technique in the conventional LSCM compared to the Bridgman-type furnace is that the temperature gradient at the solid/liquid interface is not constant but a function of pool radius. From a geometric point of view, the larger the fraction of solid rim $(Z)$, the higher the rate of heat extraction and therefore the higher the temperature gradient at the solid/liquid interface as shown schematically in Fig. 1(a). Therefore, if $r_{1}>r_{2} \Rightarrow G_{1}^{r}<G_{2}^{r}$, where $r$ is the pool radius and $G^{r}$ is the temperature gradient at the solid/liquid interface. The ellipsoidal shape of the microscope chamber concentrates the 


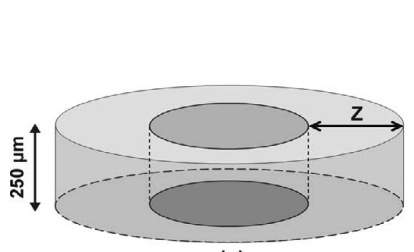

(a)

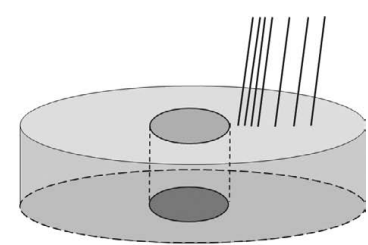

(b)

Fig. 1. Contributing parameters to the temperature gradient at the solid/liquid interface in the concentric solidification technique: (a) fraction of solid rim, $\mathrm{Z}$ and (b) the beam density.

light beam (generated by the heat source, a $1.5 \mathrm{KW}$ Halogen lamp) at the center of the sample discs. The highest beamdensity is located at the disc center and the lowest beamdensity at the disc edge, as shown in Fig. 1(b). Consequently, samples are heated un-evenly across the disc radius. Hence, a temperature gradient is created at the solid/liquid interface and it cannot be independently controlled. Another characteristic of the concentric solidification technique is that there is a continuous build-up of solute in the liquid ahead of the progressing solid/liquid interface ${ }^{3)}$ which is particularly significant at smaller pool radii.

In an attempt to overcome the difficulties outlined above, we developed an experimentally-aided approach to simulate the solidification of steel using the phase-field code MICRESS $^{\circledR}$. 4) The simulations were aimed at the quantitative prediction of microstructural development and solute segregation, while laser-scanning confocal microscopy rendered input data for the phase-field modelling study. Initially a simplified "preliminary simulation" was developed by establishing an equilibrium two-phase microstructure and a stationary planar solid/liquid interface. In order to produce more realistic simulations of microstructural developments, the solid/liquid interfacial energy and the Gibbs Thomson coefficient of the alloy under investigation were determined experimentally and used as input parameters in the simulation. The solid/liquid interfacial energy and the Gibbs Thomson coefficient were determined by analyzing the shape of the grain boundary groove at the solid/liquid interface, observed in real-time and at temperature in a laserscanning confocal microscope as will be shown below. The solid/liquid interface velocity of the solidifying alloy during the initial transient stages of solidification was determined experimentally by using a concentric solidification technique. ${ }^{2)}$ Subsequently, the interface mobility boundary condition in the simulation was set so as to predict the experimentally determined interface kinetics at the initial transient stages of solidification. Figure 2 schematically shows the approach outlined above, which was utilized to simulate the microstructural development during solidification of the steel under investigation.

Once the simulation incorporating the pertaining surface energies and the Gibbs-Thomson coefficient was formulated and verified, it was possible to extend the processing parameters of cooling rate and temperature gradient to values well beyond the experimental limitations of the LSCM and hence, to predict microstructural development at the high casting speeds encountered in modern operational continuous casting practice. The resulting microstructures predicted under such circumstances were qualitatively and quantitatively analyzed and will be discussed below.

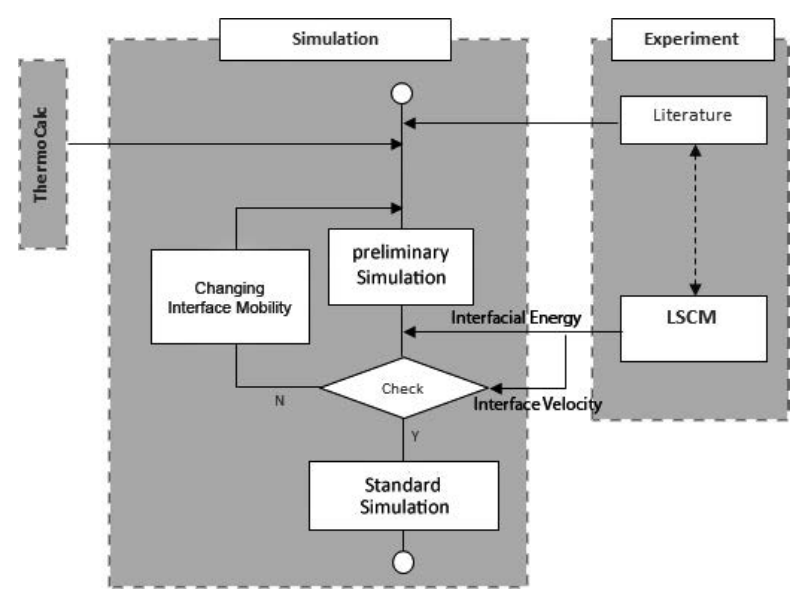

Fig. 2. Schematic illustration of the approach used to simulate the microstructural development during solidification.

\section{Multi-phase-field Model}

The simulations were performed using the commercially available MICRESS ${ }^{\circledR}$ code, which essentially implements a multi-phase-field model ${ }^{5)}$ and which has been applied to binary $^{6)}$ and multi-component systems. ${ }^{7)}$ The MICRESS $^{\circledR}$ code has been previously employed to address microstructural formation during solidification of steel..$^{8-11)}$ There is no need to describe the MICRESS ${ }^{\circledR}$ code in detail, but in the context of the present discussion, it is pertinent to briefly describe the underpinning principles of the code, which essentially provides a mathematical solution to the coupled phase-field and diffusion differential equations. In the phase field description each phase is described by a phase-field parameter (order parameter) which contains some physical properties of the different phases. In a multi-grain multiphase system, the phase-field approach assigns two distinct values of order parameters, $\varphi_{i}$. At a given location and time, if grain or phase $i$ is present: $\varphi_{i}=1$. Conversely, if grain or phase $i$ is not present at a given location and time and at an interface between grains: $\varphi_{i}=0$. A system consisting of $N$ grains or phases is thus described by a set of $N$ order parameters, $\varphi_{i}(r, t)$. Phase-field equations are solved by a set of equations similar to Eq. (1).

$$
\begin{aligned}
& \varphi_{i}(r, t)=\sum_{j} \mu_{i j} \\
& {\left[\sigma_{i j}\left(\varphi_{i} \nabla^{2} \varphi_{j}-\varphi_{j} \nabla^{2} \varphi_{i}+\frac{\pi^{2}}{2 \eta^{2}}\left(\varphi_{i}-\varphi_{j}\right)\right)+\frac{\pi}{\eta} \sqrt{\varphi_{i} \varphi_{j}} \Delta S_{i j} \Delta T_{i j}\right] .}
\end{aligned}
$$

The first group of terms in Eq. (1) incorporates the interfacial energy between phases $i$ and $j, \sigma_{i j}$, (this is to include the influence of curvature on microstructural development). The second group of terms describe the chemical thermodynamics of the system and incorporates $\eta$, the thickness of the interface, $\Delta S_{i j}$, the entropy of fusion of the alloy and $\Delta T_{i j}$, the equilibrium undercooling. $\mu_{i j}$ is the interface mobility and $\mu_{i j} \times \sigma_{i j}$ is defined as the kinetic coefficient. Subsequently, a set of phase-field equations are solved at specific time frames, by minimizing the total free energy of the system. Solute diffusion is combined with the free energy function in order to determine the kinetics of the phase transformation. The phase field equations are therefore coupled with 
the diffusion equation. Diffusion of element $\mathrm{A}$ in a system consisting of $\alpha$ and $\beta$ phases is expressed as:

$$
\frac{\partial x^{A}}{\partial t}=\nabla\left[\phi_{i} D_{\alpha}^{A} \nabla x_{\alpha}^{A}+\left(1-\phi_{i}\right) D_{\beta}^{A} \nabla x_{\beta}^{A}\right]
$$

$D_{\alpha}^{A}$ is diffusivity of the element A in phase $\alpha$ and $D_{\beta}^{A}$ the diffusivity of element $\mathrm{A}$ in phase $\beta$. The interface is the superposition of two bulk phases (or 3 bulk phases at a triple-point). Within the interface region, the total composition is split between the compositions of the two phases:

$$
c^{\text {total }}=\phi_{\alpha} c_{\alpha}+\phi_{\beta} c_{\beta}
$$

The split of total composition $\left(c^{\text {total }}\right)$ into phase compositions is done with the aid of a parallel tangent construction based on the Gibbs-energies of the bulk phases. ${ }^{7}$

\section{Simulations}

Figure 3 shows the position of the simulation domain relative to the concentric solidification experiment in the confocal microscope. In-situ observations of solidification in the confocal microscope are made in two-dimensions and are recorded in real-time digital movie format. It is important to emphasize that inherent features of the concentric solidification experiment are that there is essentially no temperature gradient in the thickness direction when the sample thickness is less than $250 \mu \mathrm{m}^{3)}$ and that observations on the surface are representative of bulk behavior. ${ }^{3)}$ Hence, the solidification simulations were conducted in two-dimensions.

In order to ensure that the concentration of the alloy far away from the moving interface remains equal to the alloy initial composition, the solidification front in the simulation was tracked by using a moving-frame boundary condition. This moving-frame boundary condition ensures that a constant distance is maintained between the tip of the largest dendrite and the top of the simulation domain. Additionally, in the simulation the temperature gradient is kept constant to produce a solidification condition similar to that of a directional solidification experimental technique. An optimum size was chosen for the simulation domain so as to balance the numerical errors occurring by using a coarse grid spacing and the increased computational times required when fine grid spacing are used. The selected simulation domain was 800 phase-field grid cells high and 400 cells

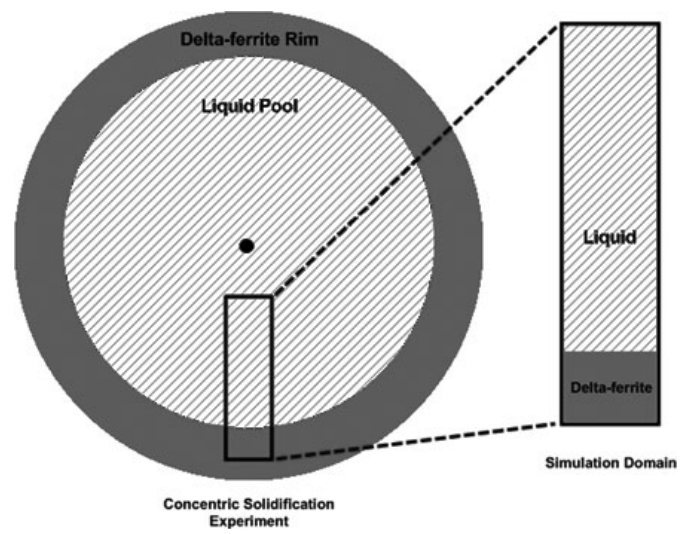

Fig. 3. Schematic representation of the simulation domain selection and its correspondence to the concentric solidification experiment. wide with a grid size of $1 \mu \mathrm{m}$. Solidification simulations were conducted on a low-carbon steel containing $0.05 \mathrm{wt} . \%$ carbon. The equilibrium solute concentrations were entered into the code by linearizing the phase-boundary lines in the $\mathrm{Fe}-\mathrm{C}$ phase diagram, which was calculated using ThermoCalc ${ }^{\mathrm{TM}}$ software. The diffusion coefficients of carbon incorporated in the simulation of Fe-C alloys, were $2.0 \times 10^{-8} \mathrm{~m}^{2} \cdot \mathrm{s}^{-1}$ and $3.95 \times 10^{-9} \mathrm{~m}^{2} . \mathrm{s}^{-1}$ in liquid and in delta-ferrite respectively. ${ }^{12)}$ In order to establish an equilibrium condition as shown in Fig. 4, carbon concentrations in the liquid and delta-ferrite phases at a given interface temperature $\left(T_{i}\right)$ were extracted from ThermoCalc ${ }^{\mathrm{TM}}$. The equilibrium fractions of solid and liquid phases at $T_{i}$ temperature were calculated using the lever rule and subsequently entered into the simulation.

Having defined the temperature of the interface $\left(T_{i}\right)$, the temperature at the bottom of the simulation domain $\left(T_{B}\right)$ was calculated under a given temperature gradient across the simulation domain $(G)$. By entering equilibrium values of the carbon concentration in the solid $\left(X_{S}^{C}\right)$ and the liquid $\left(X_{L}^{C}\right)$ at a given interface temperature $\left(T_{i}\right)$, the fractions of solid and liquid as well as the temperature at the bottom of the simulation domain could be established under equilibrium conditions. Consequently, the planar interface remained stationary at a given temperature gradient $(G)$ prior to cooling. Table 1 shows the thermodynamic data extracted from ThermoCalc ${ }^{\mathrm{TM}}$ and the calculated temperature at the bottom of the domain for establishing an equilibrium two-phase microstructure with a planar solid/liquid interface at $\mathrm{t}=0 \mathrm{~s}$ under a given temperature gradient.

The boundary conditions for the phase-field boundaries and concentration fields are different at either side of the simulation domain. For a realistic simulation, periodic boundary conditions were used on the right and left side in
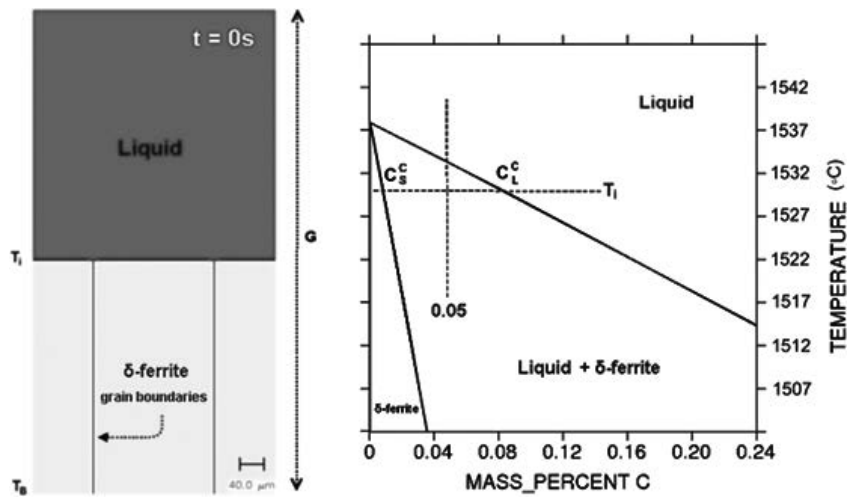

Fig. 4. An initial planar solid/liquid interface in the Fe-0.05 wt.\% $\mathrm{C}$ system, showing three delta-ferrite grains in contact with liquid.

Table 1. Thermodynamic data required to establish an equilibrium two-phase microstructure with a planar solid/liquid interface at a given temperature gradient, $\mathrm{G}$.

\begin{tabular}{ccccccccc}
\hline$T_{i}$ & $C_{S}^{C}$ & $C_{L}^{C}$ & Fraction \\
$(\mathrm{K})$ & $(\mathrm{wt}) \%$ & $(\mathrm{wt}) \%$ & of solid & of liquid & $\begin{array}{c}\text { Dength } \\
(\mu \mathrm{m})\end{array}$ & $\begin{array}{c}\mathrm{G} \\
(\mathrm{K} / \mathrm{mm})\end{array}$ & $\begin{array}{c}T_{B} \\
(\mathrm{~K})\end{array}$ \\
\hline & & & & & & 2 & 1802.4 \\
1803.2 & 0.014 & 0.083 & 0.484 & 0.515 & 800 & 20 & 1795.4 \\
& & & & & & 100 & 1764.4 \\
\hline
\end{tabular}


both phase-field parameter and concentration fields. At the top and bottom of the simulation domain, insulation phasefield and concentration field boundary conditions were used.

\subsection{Experimental Determination of Boundary Condi- tions}

In-situ observations in a laser-scanning confocal microscope (LSCM) were used to experimentally determine the solid/liquid interfacial energy of the alloy under investigation by extracting information about the geometry of the grain boundary groove at the solid/liquid interface. Details of the solidification experiments and the microscope are described elsewhere. ${ }^{2)}$ Engin et al. have shown ${ }^{13)}$ that the solid/liquid interfacial energy can be calculated from the shape of grain boundary at the solid/liquid interface. Assuming an isotropic solid/liquid interfacial energy when a planar grain boundary intersects a planar interface, the solid/liquid interfacial energy can be calculated from the Gibbs-Thomson equation for a curved interface with isotropic solid/liquid interfacial energy, Eq. (4):

$$
\Gamma=r \Delta T_{r}=\frac{\sigma_{S L}}{\Delta S^{*}}
$$

$\Delta T_{r}$ is the curvature undercooling, $\Delta S^{*}$ the entropy of fusion per unit volume, $\sigma_{S L}$ the interfacial energy between solid and liquid, $\Gamma$ is the Gibbs-Thomson coefficient and $r$ the radius of the grain boundary groove as shown in Fig. 5 .

In order to assess the feasibility of Gibbs-Thomson coefficient and the solid/liquid interfacial energy measurements from the shape of the grain boundary, in-situ and at the temperature, a low-carbon $\mathrm{Fe}-\mathrm{C}$ alloy with minimum trace elements was produced in a laboratory scale vacuum induction melting furnace.

Table 2 shows the chemical composition of this alloy, which is comparable with the composition of commercially available pure iron. The measurements where subsequently compared to the Gibbs-Thomson coefficient and the solid/liquid interfacial energy of pure iron, available in the literature. ${ }^{12)}$

Cylindrical samples of $9 \mathrm{~mm}$ diameter and $250 \mu \mathrm{m}$ thickness were cut into thin discs using an Accutum 3500 diamond cutting machine and ground on 800, 1200,2400 and

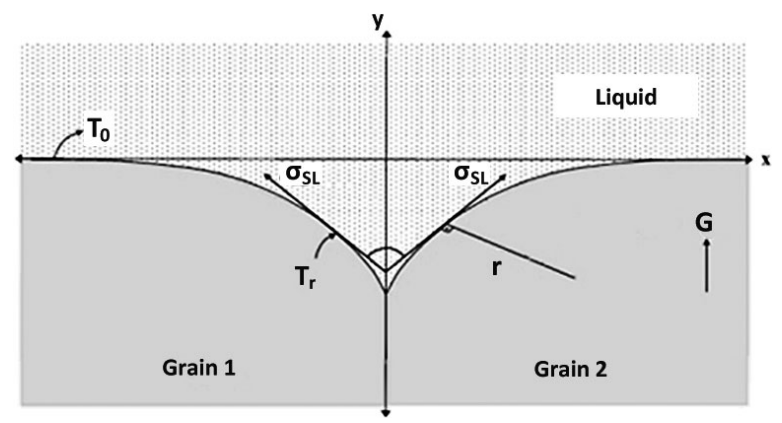

Fig. 5. Equilibrium grain boundary groove at a solid/liquid interface. $^{13)}$

Table 2. Chemical composition of the alloy.

\begin{tabular}{ccccccccc}
\hline \multicolumn{8}{c}{ Elements, wt $\%$} \\
\hline $\mathrm{C}$ & $\mathrm{Cu}$ & $\mathrm{Mn}$ & $\mathrm{P}$ & $\mathrm{S}$ & $\mathrm{Cr}$ & $\mathrm{Mo}$ & $\mathrm{Ni}$ & $\mathrm{Al}$ \\
0.0500 & $<0.002$ & $<0.01$ & 0.002 & 0.002 & $<0.002$ & $<0.002$ & $<0.002$ & 0.016 \\
\hline
\end{tabular}

4000 abrasive papers before being polished by standard metallographic techniques using 6 and $1 \mu \mathrm{m}$ diamond powder. Disc-shaped samples were inserted into the microscope chamber and heated at a rate of $100 \mathrm{~K} / \mathrm{min}$ to $1573 \mathrm{~K}$, under an ultra-high purity argon atmosphere. The heating rate was then reduced to $1 \mathrm{~K} / \mathrm{min}$ to establish a liquid pool in the center of the specimens. When the liquid pool reached a radius of $3.8 \pm 0.1 \mathrm{~mm}$, the temperature was kept constant for about 2 minutes until a stable liquid pool was formed. The size of the pool and the temperature were then precisely measured and recorded as a reference. The sample temperature was then changed to $1 \mathrm{~K}$ below the reference temperature and subsequently to $1 \mathrm{~K}$ above the reference temperature while the corresponding pool sizes were measured in each case. The temperature gradient at the solid/liquid interface for a pool size of $3.8 \pm 0.1 \mathrm{~mm}$ was then determined from the slope of the curve as shown in Fig. 6 as $7.8 \mathrm{~K} / \mathrm{mm}$.

Figure 7(a) shows a grain boundary groove at the solid/ liquid interface under equilibrium conditions in the low-carbon steel under investigation. The curvature radius $(r)$ and the curvature depth $(h)$ of the delta-ferrite grains were measured and presented in Table 3. The curvature undercooling $\left(\Delta T_{r}\right)$ was calculated from the curvature depth $(h)$ and the

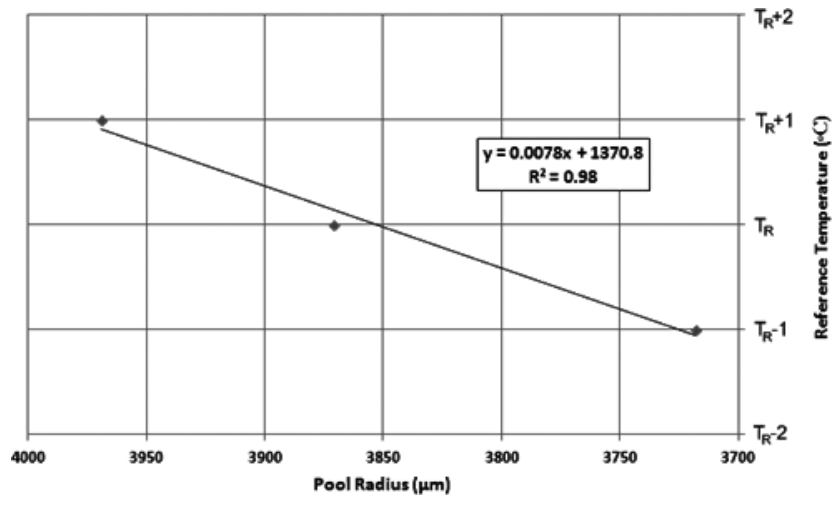

Fig. 6. Measurement of the temperature gradient at the solid/liquid interface.

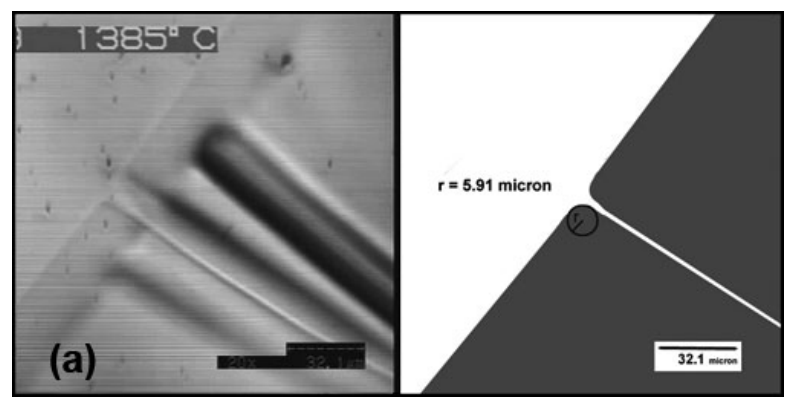

Fig. 7. Grain boundary groove at equilibrium at the solid/liquid interface in $\mathrm{Fe}-0.05 \mathrm{wt} . \% \mathrm{C}$ alloy. The image on the right hand side is a schematic representation of the original image.

Table 3. Calculation of Gibbs-Thomson coefficients and the solid/ liquid interfacial energies in the low carbon steel.

\begin{tabular}{ccccccc}
\hline $\begin{array}{c}\text { Composition } \\
(\mathrm{wt}) \%\end{array}$ & $\begin{array}{c}\mathrm{h} \\
(\mathrm{mm})\end{array}$ & $\begin{array}{c}\Delta \mathrm{T}_{\mathrm{r}} \\
(\mathrm{K})\end{array}$ & $\begin{array}{c}\mathrm{r} \\
(\mathrm{mm})\end{array}$ & $\begin{array}{c}\Gamma \\
(\mathrm{Kmm})\end{array}$ & $\begin{array}{c}\Delta \mathrm{S}^{*} \\
(\mathrm{~J} / \mathrm{m} 3 \mathrm{~K})\end{array}$ & $\begin{array}{c}\sigma_{\mathrm{SL}} \\
\left(\mathrm{J} / \mathrm{mm}^{2}\right)\end{array}$ \\
\hline Fe-0.05C & $4.1 \mathrm{E}-03$ & $3.2 \mathrm{E}-02$ & $5.91 \mathrm{E}-03$ & $1.89 \mathrm{E}-04$ & $1.07 \mathrm{E}-06$ & $2.02 \mathrm{E}-07$
\end{tabular}




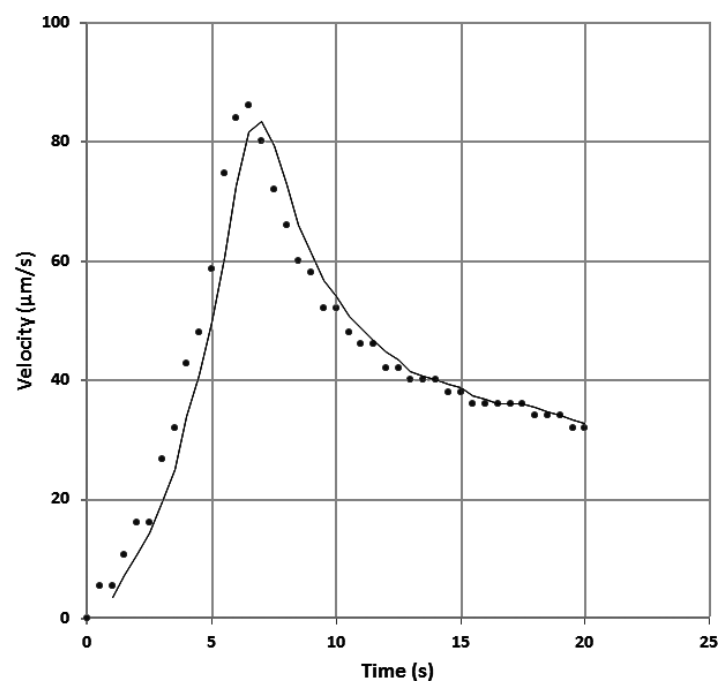

Fig. 8. Model predictions of the solid/liquid interface velocity from the commencement of cooling at a time when the interface was planar to the initiation and growth of cellular grains. Cooling rate: $2 \mathrm{~K} / \mathrm{min}$.

temperature gradient at the interface $(G)$ using Eq. (5):

$$
\Delta T_{r}=G^{*} h
$$

The value for the entropy of fusion of delta-ferrite steel was extracted from the literature: ${ }^{12)} \Delta \mathrm{S}^{*}=1.07 \times 10^{-6}(\mathrm{~J} / \mathrm{m} 3 \mathrm{~K})$.

Table 3 shows the calculation of Gibbs-Thomson coefficient and the solid/liquid interfacial energy in the low carbon steel.

The calculated interfacial energy of low carbon steel is almost the same as the solid/liquid interfacial energy of pure iron of $2.04 \times 10^{-7} \mathrm{~J} / \mathrm{mm}^{2}$ reported in the literature. ${ }^{12)}$ The minimal difference could be attributed to the fact that, although the addition of carbon does not change the interfacial energy of pure iron, carbon potentially raises the activity of sulphur in steel which consequently reduces the interfacial energy of the system. ${ }^{14)}$ The measured Gibbs-Thomson coefficient for delta-ferrite in low-carbon steel is also in good agreement with the reported Gibbs-Thomson coefficient $^{12)}$ of $1.91 \times 10^{-4} \mathrm{Kmm}$. It is interesting to note that the present approach can also be applied to other alloying systems as long as it is possible to use the concentric solidification technique. For instance, the influence of copper additions on the solid/liquid interfacial energy and the Gibbs Thomson coefficient of the studied low-carbon steel were assessed. For this purpose, precisely measured amounts of copper were added to the melt pool and the shape of the grain boundary groove at the solid/liquid interface was analyzed. However, the investigation of the effect of copper is beyond the scope of the current study.

In the concentric solidification technique and the present solidification simulation, the interface velocity is controlled by the rate of cooling. Figure $\mathbf{8}$ shows the model predictions of the solid/liquid interface velocity from the commencement of cooling at a time when the interface was planar to the initiation and growth of cellular grains. As expected, a transient regime is present following the commencement of cooling where the interface velocity initially increases. The Initial increase of the interface velocity occurs before the carbon concentration profile in liquid ahead of the interface
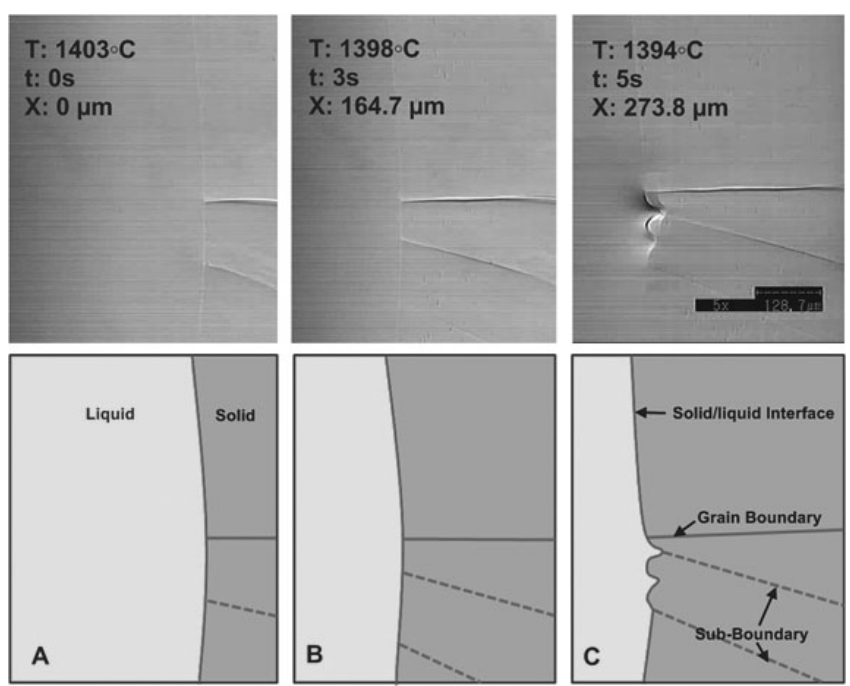

Fig. 9. Solid/liquid interface in a low-carbon steel cooled at a rate of $100 \mathrm{~K} / \mathrm{min}$, at the onset of planar to cellular transition. The bottom row shows schematics of the images at the top row.

is stabilized. The increased velocity regime is followed by a second transient period where the interface velocity decays as the carbon concentration build up in liquid phase before the velocity attains a constant value.

In order to calibrate this initial transient regime of the interface velocity in the simulations, the interface mobility, which is a boundary condition in the simulation was adjusted so to reproduce of the experimentally-measured interface velocities during the planar to cellular transition.

A liquid pool with a diameter of $3.00 \pm 0.50 \mathrm{~mm}$ was established upon heating a sample and the average temperature gradient at the solid/liquid interface for pool sizes between $3.5 \mathrm{~mm}$ and $2.5 \mathrm{~mm}$ was measured in similar vein to the experiment outlined in Fig. 6, which was approximately $2.0 \mathrm{~K} / \mathrm{mm}$. Samples were then cooled at rates of 100 $\mathrm{K} / \mathrm{min}$ or $520 \mathrm{~K} / \mathrm{min}$ respectively while the solid/liquid interface was accurately tracked. Interface velocities were subsequently measured at the onset of planar to cellular interface transition and at a distance of less than $300 \mu \mathrm{m}$ so as to minimize the effect of a varying temperature gradient across the disc-shaped sample. Figure 9 is an example of the interface tracking at the onset of planar to cellular transition, where $X$ is the interface location in the different time frames.

The same cooling rates and temperature gradients were then used in the solidification simulation and the interface velocity at the onset of the planar to cellular transition predicted. The interface mobility between liquid and the delta-ferrite phase was subsequently calibrated in such a way to as to reproduce the experimentally-observed interface velocities during the planar to cellular transition. Good agreement between the simulation and experiment was found when an interface mobility of $1.0 \times 10^{4} \mathrm{~mm}^{4} /(\mathrm{Js})$ was used. Figure 10 shows (a) the solid/liquid interface position and interface velocity at the occurrence of the planar to cellular transition and (b) a comparison between the experimentally measured and calculated interface velocities. 

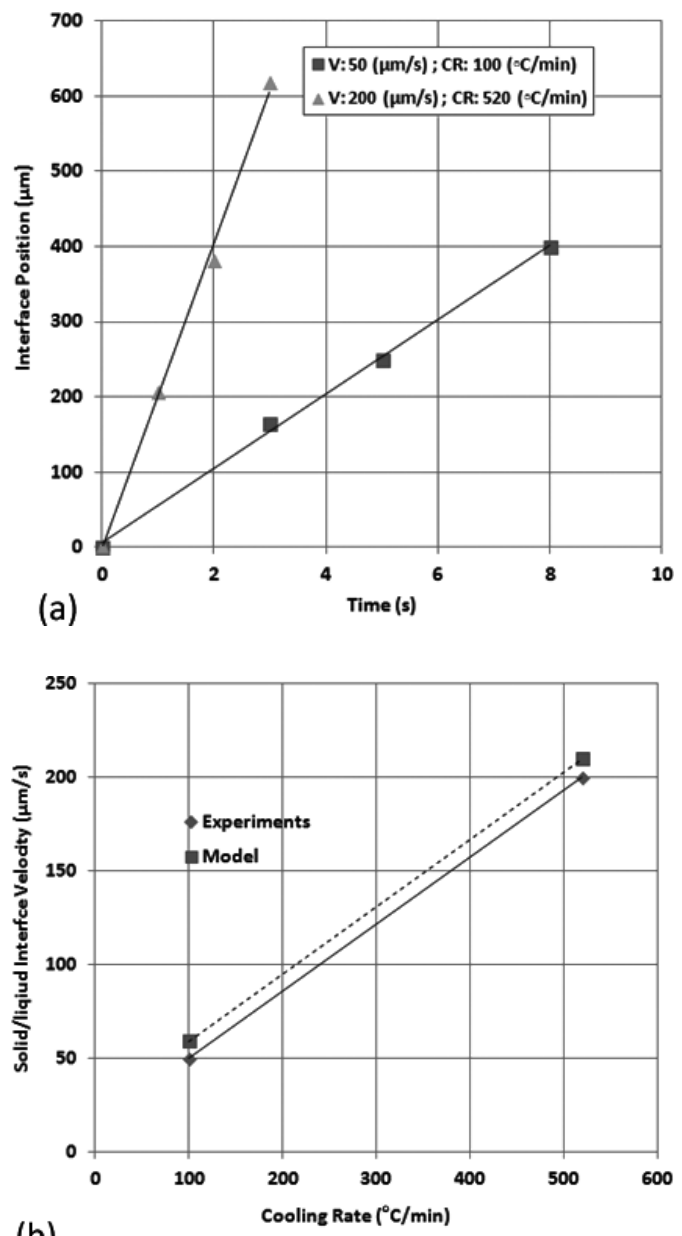

(b)

Fig. 10. (a) Interface position and the average interface velocity at the occurrence of planar to cellular transition and (b) a comparison between the experimentally measured and calculated interface velocities.

\subsection{Microstructural Simulations}

The influence of cooling rate and temperature gradient on the development of solidification microstructures were predicted by implementing the phase-field code MICRESS ${ }^{\circledR}$. At varying cooling rates and under a constant temperature gradient of $2 \mathrm{~K} / \mathrm{mm}$, the initial planar interface became unstable and developed perturbations as depicted in Fig. 11.

After 20 seconds, at a constant temperature gradient of 2 $\mathrm{K} / \mathrm{mm}$ and a under cooling rate of $20 \mathrm{~K} / \mathrm{mm}$, the planar interface developed into a cellular structure as shown in Fig. 11(a). At cooling rates of $200 \mathrm{~K} / \mathrm{min}$ and $1000 \mathrm{~K} / \mathrm{min}$ respectively, a solidification microstructure consisting of an array of dendrites is predicted as illustrated in Figs. 11(b) and 11(c). Due to the increased interface velocity at higher cooling rates, the solidification front reaches the $1 \mathrm{~mm}$ distance after 1.5 seconds at a cooling rate of $200 \mathrm{~K} / \mathrm{min}$ as shown in Fig. 11(b) while, this distance is reached after only 0.5 seconds at a cooling rate of $1000 \mathrm{~K} / \mathrm{min}$ as shown in Fig. 11(c). Carbon concentration profiles at the dendrite tips of Figs. 11(b) and 11(c) are shown in Fig. 12.

At a cooling rate of $200 \mathrm{~K} / \mathrm{min}$, the carbon concentration gradient at the dendrite tip, shown in Fig. 12(a) is 7.0 wt. $\% \mathrm{C} / \mu \mathrm{m}$, while at a cooling rate of $1000 \mathrm{~K} / \mathrm{min}$, this concentration gradient is $18.3 \mathrm{wt} . \% \mathrm{C} / \mu \mathrm{m}$. The temperature gradient cannot be independently controlled in the concentric

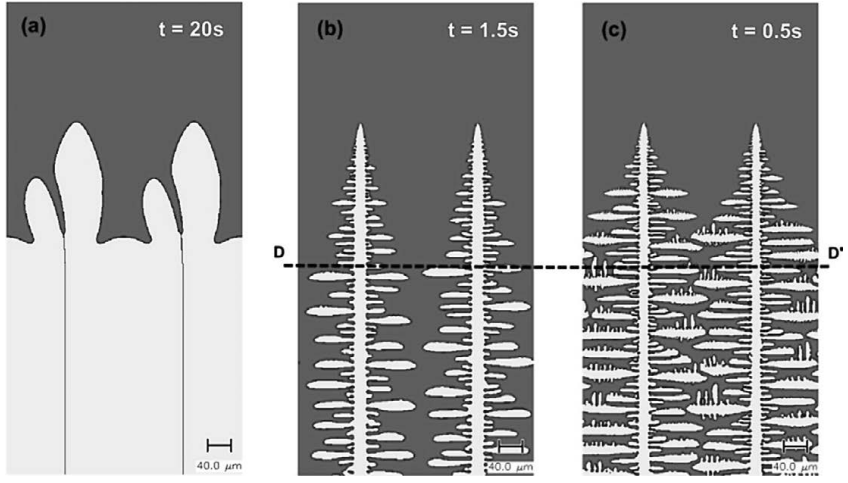

Fig. 11. Effect of cooling rate on microstructural development during solidification. The temperature gradient is constant $2 \mathrm{~K} / \mathrm{mm}$, and the cooling rates are (a) $20 \mathrm{~K} / \mathrm{min}$, (b) 200 $\mathrm{K} / \mathrm{min}$ and (c) $1000 \mathrm{~K} / \mathrm{min}$. The section DD' line is $1 \mathrm{~mm}$ from the initial planar solid/liquid interface.
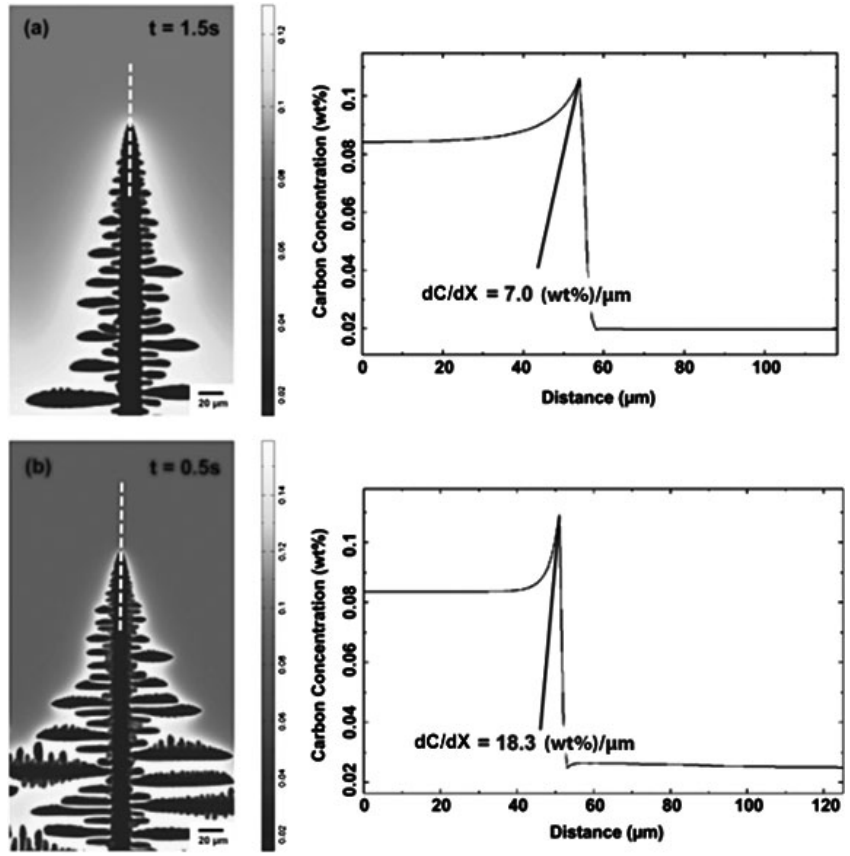

Fig. 12. Carbon concentration profiles at dendrite tips following cooling rates of (a) $200 \mathrm{~K} / \mathrm{min}$ and (b) $1000 \mathrm{~K} / \mathrm{min}$. The temperature gradient is constant, $2 \mathrm{~K} / \mathrm{mm}$.

solidification experiment, however it can be treated as an independent parameter in the MICRESS ${ }^{\circledR}$ simulations, and hence, the influence of temperature gradient on microstructural development can be studied in detail. Solidification microstructures after 20 seconds under a temperature gradient of (a) $2 \mathrm{~K} / \mathrm{mm}$, (b) $20 \mathrm{~K} / \mathrm{mm}$ and (c) $100 \mathrm{~K} / \mathrm{mm}$ respectively and under a constant cooling rate of $200 \mathrm{~K} / \mathrm{min}$ are presented in Fig. 13. The stabilizing effect of temperature gradient on the development of a planar solid/liquid interface development is evident since the interface remained planar under a temperature gradient $100 \mathrm{~K} / \mathrm{mm}$. The primary dendrite arm spacing is reduced when the temperature gradient is increased from $2 \mathrm{~K} / \mathrm{mm}$ to $20 \mathrm{~K} / \mathrm{mm}$. A stable planar solid/liquid interface is established under a temperature gradient of $200 \mathrm{~K} / \mathrm{min}$.

Figure 14 shows the carbon concentration profiles at the 


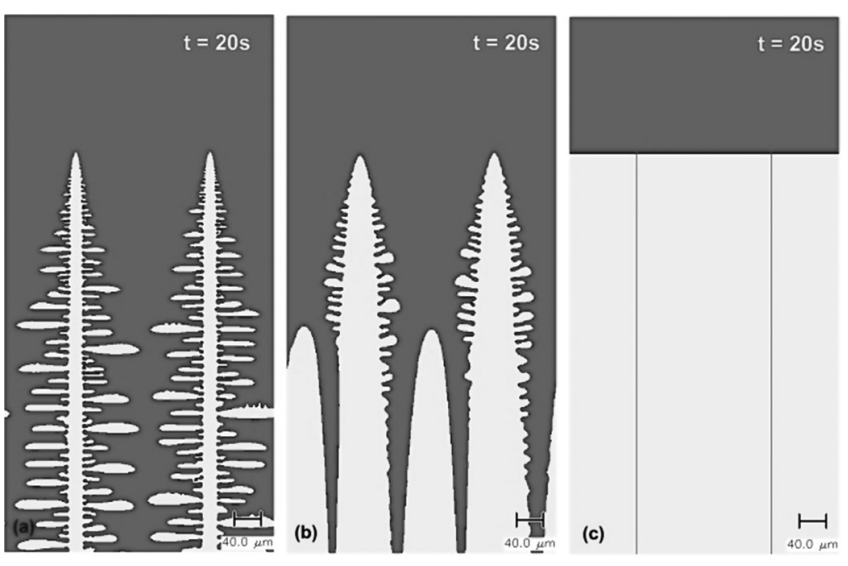

Fig. 13. Effect of temperature gradient on solidification microstructure. Temperature gradients are: (a) $2 \mathrm{~K} / \mathrm{mm}$, (b) $20 \mathrm{~K} / \mathrm{mm}$ and (c) $100 \mathrm{~K} / \mathrm{mm}$. Cooling rate is constant, $200 \mathrm{~K} / \mathrm{min}$ and the simulation time is 20 seconds.
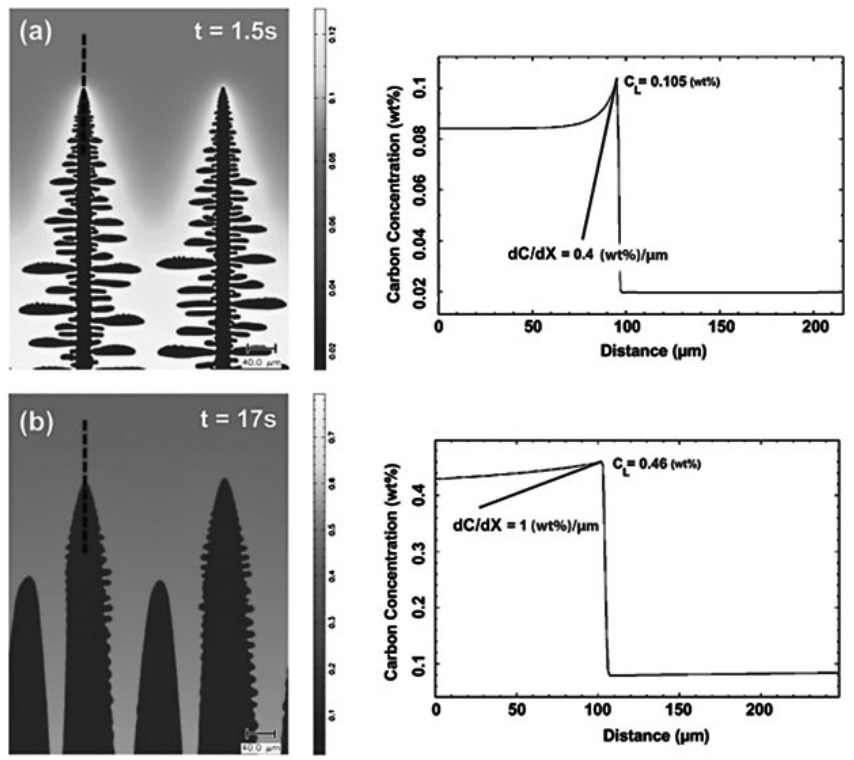

Fig. 14. Carbon pile-up in the liquid at dendrite tips under temperature gradients of (a) $2 \mathrm{~K} / \mathrm{mm}$ and (b) $20 \mathrm{~K} / \mathrm{mm}$ respectively. The cooling rate: $200 \mathrm{~K} / \mathrm{min}$.

dendrite tips $1 \mathrm{~mm}$ ahead of the initial planar interface at a cooling rate of $200 \mathrm{~K} / \mathrm{min}$ and temperature gradients (a) 2 $\mathrm{K} / \mathrm{mm}$ and (b) $20 \mathrm{~K} / \mathrm{mm}$ respectively.

Figure 14 shows that increased temperature gradients lead to increased carbon pile-up and carbon concentration gradients in the liquid ahead of the dendrite tips. At a cooling rate of $200 \mathrm{~K} / \mathrm{min}$ and under a temperature gradient of $2 \mathrm{~K} / \mathrm{mm}$, the carbon concentration in the liquid ahead of dendrite tips is $0.10 \mathrm{wt} . \% \mathrm{C}$ and the concentration gradient in the liquid is $0.4 \mathrm{wt} . \% \mathrm{C} / \mu \mathrm{m}$. Increasing the temperature gradient to 20 $\mathrm{K} / \mathrm{mm}$ results in a carbon pile up of $0.46 \mathrm{wt} . \% \mathrm{C}$ and a concentration gradient of $1 \mathrm{wt} . \% \mathrm{C} / \mu \mathrm{m}$ in the liquid.

\section{Conclusions}

An in-situ study of solidification of a low-carbon steel within a laser-scanning confocal microscope rendered input data for a phase-field modelling study aimed at the quantitative prediction of microstructural development and solute segregation. The solid/liquid interfacial energy and the Gibbs-Thomson coefficient of the alloy was experimentally measured by analysing the shape of the grain boundary groove at the solid/liquid interface. This in-situ experimental technique can be extended to the study of other alloying systems, for example copper-containing $\mathrm{Fe}-\mathrm{C}$ alloys. The interface mobility, a boundary condition in the phase-field model, was adjusted to reproduce the experimentally measured velocities of solidification fronts at the onset of interface instability. The interface mobility thus determined was then used in further calculations. Microstructural development during solidification and carbon segregation in liquid ahead of dendrite tips were assessed as functions of cooling rate and temperature gradient. At a fixed temperature gradient, an increasing cooling rate increased the carbon concentration gradient in the liquid ahead of the dendrite tips and the tendency towards planar to cellular to dendritic growth. At a constant cooling rate, an increasing temperature gradient increased the carbon concentration gradient in the liquid ahead of the dendrite tips, but reduced the tendency towards planar to cellular to dendritic transition.

\section{Acknowledgement}

We wish to thank the University of Wollongong for the provision of laboratory facilities and permission to publish our work. We gratefully acknowledge the financial support of the ARC through Discovery Grant DP0879319. We also wish to express our thanks to Prof. Jilt Sietsma and Dr. Dominic Phelan for many fruitful discussions.

\section{REFERENCES}

1) H. Chikama, H. Shibata, T. Emi and M. Suzuki: Mater. Trans. JIM, 37 (1996), 620.

2) M. Reid, D. Phelan and R. Dippenaar: ISIJ Int., 44 (2004), 565.

3) S. Niknafs, D. Phelan and R. Dippenaar: J. Microsc., 249 (2013), 53.

4) www.Micress.de, the version 5.25 of the software was used.

5) I. Steinbach, F. Pezzolla, B. Nestler, M. Seeßelberg, R. Prieler, G. J. Schmitz and J. L. L. Rezende: Physica D, 94 (1996), 135.

6) J. Tiaden, B. Nestler, H. J. Diepers and I. Steinbach: Physica D, 115 (1998), 73.

7) J. Eiken, B. Böttger and I. Steinbach: Phys. Rev. E, 73 (2006), 066122.

8) K. Nakajima, M. Apel, J. Eiken and I. Steinbach: Acta Mater., 54 (2006), 3665.

9) B. Böttger, M. Apel, B. Santillana and D. G. Eskin: IOP Conf. Series, Mater. Sci. Eng., 33 (2012), 012107.

10) B. Böttger, M. Apel, B. Santillana and D. G. Eskin: Metall. Mater. Trans. A, 44 (2013), 3765.

11) P. Schaffnit, C. Stallybrass, J. Konrad, A. Kulgemeyer and H. Meuser: Int. J. Mater. Res., 101 (2010), 549.

12) W. Kurz and D. J. Fisher: Fundamentals of Solidification, 4th rev. ed., Trans Tech Publication LTD, Durnten, Zuerich, (1998), 293.

13) S. Engin, U. Böyük and N. Maraslı: Current Appl. Phys., 11 (2011), 1060 .

14) W. T. Lankford, N. L. Samways, R. F. Craven and H. E. McGannon: The Making, Shaping and Treating of Steel, 10th ed., Association of Iron and Steel Engineers, Pittsburgh, (1985), 412. 